\title{
Los procesos industriales sostenibles y su contribución en la prevención de problemas ambientales
}

\section{RESUMEN}

El presente artículo tiene por objeto dar a conocer una nueva concepción sobre los procesos industriales, los denominados procesos industriales sostenibles. Estos son utilizados en las plantas industriales de empresas responsables y competitivas, para elaborar productos químicos. Estos procesos innovadores deben incorporar en su diseño etapas que sean eficientes, generen menos residuos y desechos, no contaminen el ambiente y utilicen sustancias que sean inocuas, así como los productos y subproductos elaborados por ellas. Estos procesos deben ser seguros, tanto internamente (para los trabajadores), como externamente (para las comunidades y ecosistemas) y deben contribuir al Desarrollo Sostenible, garantizando el uso eficiente de recursos naturales y propiciando el empleo de recursos renovables. Un ejemplo, es la producción de pulpa de celulosa a nivel latinoamericano (Brasil, Argentina y Uruguay), pero que con un proceso industrial sostenible puede ser elaborado a nivel nacional.

Palabras clave: proceso industrial sostenible, empresa responsable y competitiva, química verde, ingeniería verde

AND THEIR CONTRIBUTION TO THE PREVENTION OF ENVIRONMENTAL PROBLEMS

\section{ABSTRACT}

This article aims to provide a new concept of industrial processes, the so-called sustainable industrial processes that are used in industrial plants and competitive companies responsible for developing chemicals. These innovative processes should incorporate in its design stages that are efficient, producing less waste and scrap, not pollute the environment, using substances which are safe, and the products and by-products produced by them. These processes must be safe, both internally (for workers) and externally (for communities and ecosystems) and must contribute to sustainable development, ensuring the efficient use of natural resources and promoting the use of renewable resources. An example is the production cellulose pulp that is produced throughout Latin America (Brazil, Argentina and Uruguay), but a sustainable industrial process can be developed nationally.

Keywords: sustainable industrial process, responsible and competitive business, green chemistry, green engineering

\section{INTRODUCCIÓN}

Los problemas ambientales relacionados con las actividades de la industria en general, y de la industria química en particular pueden ser prevenidos si es que los procesos productivos que utilizan las empresas, cumplen con una serie de requisitos y tienen como base para su diseño un conjunto de principios que deben ser tomados en cuenta por los ingenieros de procesos. Para ello es necesario introducir un nuevo paradigma, el proceso industrial sostenible, que hará posible que las empresas sean responsables (por sus actividades "limpias", seguras y sin generar problemas ambientales) y competitivas (puedan interactuar en mercados nacionales e internacionales, en las mismas condiciones con otras empresas del sector).

\section{PROCESOS INDUSTRIALES SOSTENIBLES}

Un proceso químico industrial es el conjunto de etapas que hacen posible la transformación de la materia prima e insumos en productos, subproductos, residuos y desechos; usando racionalmente la energía, y teniendo en cuenta en cada etapa las condiciones de operación que hagan posible procesos eficientes. Las etapas son actividades unitarias que pueden ser operaciones unitarias o procesos unitarios, aunque entre algunas de ellas la diferencia es muy sutil y en otras se complementan.

Los procesos químicos industriales sostenibles o procesos industriales sostenibles, son procesos también constituidos por etapas que son actividades unitarias, pero que potencian el aprovechamiento de los materiales y la energía para la producción de bienes (o productos útiles) y minimizan o eliminan la presencia de residuos y desechos -o males- (ya que dependiendo del tipo de residuo, estos pueden contribuir a la contaminación ambiental y a sus efectos).

Los procesos industriales tienen que contribuir al desarrollo sostenible, entendido como el tipo de desarrollo orientado a garantizar la satisfacción de las necesidades fundamentales de la población y elevar su calidad de vida, a través del manejo racional de los recursos naturales, propiciando su conservación, recuperación, mejoramiento y uso adecuado, de tal manera que

\footnotetext{
* Magíster en Economía del Medio Ambiente y Recursos Naturales. Ingeniero Químico. Profesor Principal. Departamento Académico de Procesos. Facultad de Química e Ingeniería Química UNMSM. E-mail: jeloayzap@yahoo.es

** Ingeniera Química. Profesora Asociada. Departamento Académico de Química Inorgánica. Facultad de Química e Ingeniería Química. UNMSM. E-mail: vickysm02@yahoo.es
} 
esta generación y las futuras tengan posibilidad de utilizarlos y disfrutarlos, sobre bases éticas y de equidad, garantizando la vida en todas sus manifestaciones.

Los principios del diseño de procesos industriales sostenibles, tienen que conjugar aspectos inherentes al diseño de procesos, minimizando el impacto ambiental y mejorando la sostenibilidad del diseño final.

El diseño sostenible en ingeniería de procesos se basa necesariamente en el diseño tradicional de ingeniería química, apoyándose además en las disciplinas como la Química Verde, la Ingeniería Verde, el diseño integrado de la cuna a la cuna, la ecología industrial y la biomimética. La integración de estas disciplinas en el panorama actual del diseño permitirá crear un marco de referencia para el desarrollo de productos, procesos y sistemas de producción, cuyos componentes no sean peligrosos, generen un estado de bienestar, consideren y respeten cada uno de los ciclos de vida de los productos que intervienen e imiten en lo posible a los sistemas naturales [3].

\subsection{Química Verde}

La Química Verde se refiere al "diseño de productos y procesos químicos que reducen o eliminan el uso y generación de sustancias peligrosas". Esta práctica se inició en los Estados Unidos tras la promulgación de la Ley de Prevención de la Contaminación de 1990. La reducción de la contaminación en la fuente, según esta ley, "es fundamentalmente diferente y más deseable que la gestión de los residuos y el control de la contaminación". Luego de la promulgación de la ley, la Oficina de Prevención de la Contaminación y Sustancias Tóxicas (OPPT) de la EPA (Agencia de Protección Ambiental de los Estados Unidos) empezó a explorar la idea de desarrollar productos y procesos químicos nuevos o mejorar los existentes, a fin de disminuir su peligrosidad. En 1991, la OPPT puso en marcha un programa modelo que otorgó, por primera vez, subsidios para proyectos de investigación que incluyeran la prevención de la contaminación en la síntesis de sustancias químicas. Desde entonces, el Programa de Química Verde de la EPA ha forjado colaboraciones con instituciones académicas, industria, otros organismos gubernamentales y organizaciones no gubernamentales para promover la prevención de la contaminación a través de la Química Verde [7].

En 1998, Paul T. Anastas y John C. Warner establecieron 12 principios que conforman la metodología de la Química Verde [3].

Figura 1. Fundamentos para el diseño de procesos industriales sostenibles.

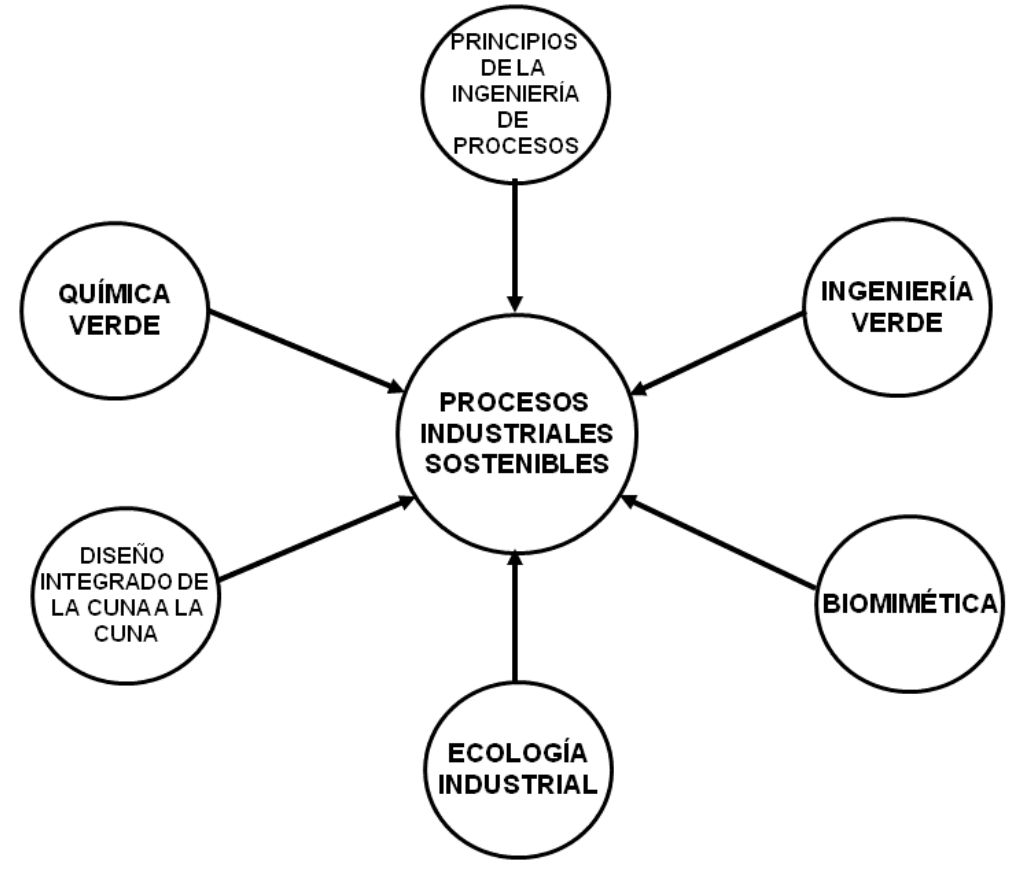

Fuente: Elaboración propia. 


\subsection{Ingeniería Verde}

La Ingeniería Verde se desarrolló como extensión de la Química Verde y tiene un alcance más amplio. David Allen y David Shonnard conciben la ingeniería verde como el "diseño, comercialización, y uso de procesos y productos, los cuales son técnica y económicamente viables, a la vez que minimizan: 1) la generación de contaminación y 2) el riesgo para la salud y el medioambiente" [4]. La Ingeniería Verde más que una disciplina nueva, está destinada a transformar las disciplinas y prácticas tradicionales de la ingeniería en otras nuevas que aumenten la sostenibilidad [3].

La Ingeniería Verde tiene 12 principios que a continuación se indican [4].

Principio 1. Los diseñadores deben esforzarse por asegurar que todas las entradas y salidas de materia y energía sean tan inherentemente inocuas como sea posible.

Principio 2. Es mejor prevenir la contaminación que tratar o limpiar el residuo ya producido.

Principio 3. Las operaciones de separación y purificación deberían diseñarse para minimizar el consumo de energía y el uso de materiales.

Principio 4. Los productos, procesos y sistemas deberían diseñarse para la maximización de la eficiencia en el uso de materia, energía y espacio.

Principio 5. Los productos, procesos y sistemas deberían estar orientados hacia la "producción bajo demanda" más que hacia el "agotamiento de la alimentación".

Principio 6. La entropía y la complejidad inherentes, deben ser consideradas como una inversión al elegir entre reutilizar, reciclar o rechazar como residuo final.

Principio 7. Diseñar para la durabilidad, no para la inmortalidad.

Principio 8. Satisfacer la necesidad, minimizar el exceso.

Principio 9. Minimizar la diversidad de materiales.

Principio 10. Cerrar los ciclos de materia y energía del proceso tanto como sea posible.

Principio 11. Diseñar para la reutilización de componentes tras el final de la vida útil del producto.

Principio 12. Las entradas de materia y energía deberían ser renovables.

\subsection{Ecología industrial}

La ecología industrial plantea una analogía entre los sistemas ecológicos naturales y la "comunidad" de plantas industriales (infraestructuras 0 instalaciones industriales). Al igual que en un ecosistema biológico, en un ecosistema industrial cada proceso debe ser visto como una parte dependiente e interrelacionada de un todo o de un sistema mayor [9]. La ecología industrial explora nuevas posibilidades para la interrelación entre empresas, como resultado de un replanteamiento de las actividades industriales y en respuesta al conocimiento cada vez más completo sobre sus impactos ambientales [2].

\subsubsection{Encadenamiento productivo entre plantas industriales}

Uno de los principales factores que afectan las condiciones ambientales de las ciudades o centros urbanos, es su cercanía a las plantas industriales (o fábricas). Las consecuencias negativas de esta localización son, entre otras, la contaminación ambiental local debido a los residuos industriales no reaprovechados y los desechos inadecuadamente gestionados, la congestión producida por el incremento del tráfico y el deterioro de la calidad de vida. En ese sentido, durante las últimas décadas, los países desarrollados y algunos países en vías de desarrollo, han empezado a ubicar sus industrias en las afueras de las ciudades o han trasladado las mismas desde el centro hacia la periferia, conformando los denominados parques industriales. Es en este contexto en que, nace el término Parque Industrial Ecoeficiente (PIE), también llamado ecoparque o parque industrial sostenible, el cual se refiere a una comunidad de plantas industriales que cooperan entre sí, compartiendo recursos, para lograr mejoras sociales y ambientales.

\subsubsection{Parques Industriales Ecoeficientes (PIE)}

En los ecosistemas naturales se pueden identificar cadenas alimenticias formadas por productores y consumidores quienes, gracias a un balanceado metabolismo, permiten que los ecosistemas permanezcan en el tiempo; es decir, sean sostenibles. Es así que, luego del análisis de los metabolismos que se observan en los sistemas ecológicos naturales, surgió la idea de implementar un PIE, que consiste en el análisis de flujos de materiales desde unas plantas industriales hacia otras plantas industriales, de diversas empresas, situadas en un lugar geográfico específico, lo que se ha denominado metabolismo industrial. Esta alternativa, que surge al conectar las fuentes de generación de residuos de un determinado producto con las fuentes de producción de otros productos, 
permite que los residuos puedan ser reutilizados, mediante lo que se denomina el encadenamiento productivo.

En el sistema planteado por la ecología industrial se busca la interacción entre cuatro grandes grupos de actores diferentes: los que extraen las materias primas de los recursos naturales, las empresas manufactureras, las empresas relacionadas con el reaprovechamiento de residuos (por ejemplo, reciclaje) y los consumidores finales.

Los parques industriales ecoeficientes son comunidades de negocios en los cuales las empresas cooperan entre sí, compartiendo sus recursos (información, materiales, residuos, recursos humanos, energía y otros) para alcanzar una mejora económica y social. A su vez se reducen los impactos sobre el medio ambiente y se obtiene de forma directa, un incremento en su competitividad, así como una reducción del consumo de recursos [5].

Independientemente de su configuración, un PIE es un grupo de plantas industriales de diversas empresas, localizadas dentro de una misma área geográfica, las cuales se dedican a la manufactura y a la prestación de servicios. Este grupo de empresas, desarrollan conjuntamente proyectos que buscan mejorar su desempeño económico y ambiental. De esta manera el trabajo coordinado les permite obtener un beneficio colectivo mayor que la suma de beneficios individuales que podría alcanzar cada empresa, optimizando únicamente su propio desempeño ambiental. Un PIE constituye una alternativa para producir limpiamente bajo parámetros competitivos, al centralizar funciones generales administrativas, logísticas, productivas, comerciales y tecnológicas; de tal manera que se logre optimizar el desempeño ambiental de las empresas participantes y disminuir costos de producción. De esta manera se logra un mayor desarrollo del concepto de ecoeficiencia, que requiere de estrategias; tales como, especialización de funciones, encadenamientos productivos, infraestructura compartida, implementación de prácticas de producción más limpia, aplicación de programas de eficiencia energética, uso eficiente de recursos, manejo unificado de la responsabilidad social y preparación conjunta para hacer frente a contingencias mediante una rápida respuesta a emergencias.

El concepto de PIE solamente se formalizó a nivel Internacional en el año 1993, por el trabajo de un equipo conformado por "Indigo Development", la Universidad de Dalhousieen Escocia y la Universidad Cornell en Estados Unidos; a partir de ello la EPA (o la Agencia de Protección Ambiental de los Estados
Unidos, USEPA) acordó un contrato con Research Triangle Institute e Indigo para extender el concepto de PIE. Los Parques Industriales Ecoeficientes surgen a nivel mundial como una alternativa de mejoramiento, tanto del desempeño ambiental de las empresas como de su desempeño económico, a través del trabajo conjunto y la interacción de las empresas vinculadas.

El parque industrial tradicional como un espacio en el cual se aglomeran empresas productivas sin ningún nivel de coordinación, debe ser superado con la implementación de parques industriales ecoeficientes. Se pueden identificar dos tipos de PIE, el primero conformado por un conjunto de empresas que tienen sus procesos encadenados, pero que realizan diferentes actividades industriales, por ejemplo el Parque Ecológico Industrial Fairfield (Baltimore, Maryland, con un excelente acceso por puerto, tren y carretera, que ha logrado la creación de un proceso industrial de "circuito cerrado") y el segundo tipo formado por empresas que realizan la misma actividad, por ejemplo, en Bogotá, Colombia, el Plan de Ordenamiento Territorial de la ciudad está buscando agrupar $80 \%$ de industriales del sector de curtiembres en el PIE del área de San Benito.

De las experiencias realizadas a la fecha es posible identificar dos grandes grupos de beneficios obtenidos al implementar un PIE:

- Lageneración de un valor agregado importante en la competitividad de las empresas participantes (cuyo beneficio colectivo es mayor que la suma de los beneficios individuales), ya que es posible el desarrollo conjunto mediante capacitación e investigación, la transferencia de tecnología, la información actualizada, el tratamiento conjunto de residuos, el uso eficiente de la materia prima e insumos, la optimización de los costos de mantenimiento).

- Los PIE permiten el ordenamiento de zonas industriales y el mejoramiento de la calidad ambiental, lo que trae como consecuencia el aumento de la calidad de vida de la población, en su conjunto.

\subsection{Biomimética aplicada al diseño de equipos}

La biomimética consiste en la aplicación de diseños naturales para resolver problemas de ingeniería, ciencia de materiales, medicina y otros campos. Un ejemplo de esto, lo han dado los investigadores de la empresa John Deere, organización que comercializa productos agrícolas y artículos para el cuidado de espacios verdes y campos de golf, quienes han aprovechado los aportes de la 
biomimética para la construcción de su tractoraraña. Esta metodología creativa, que consiste en imitar a la naturaleza para aplicarla al diseño tecnológico e industrial, ha convertido el clásico tractor unidireccional en un "insecto industrial" capaz de adaptarse a distintos terrenos, tal como lo haría una araña. De esta forma, el tractor no gana en potencia ni en complejidad tecnológica pero sí en libertad de movimientos, hecho que permite poder trabajar en zonas que hasta ahora eran impracticables por su complicada orografía. Tal como ya se percataron Leonardo da Vinci o Antoni Gaudí en su época, la naturaleza se erige de nuevo como fuente de inspiración tanto para el diseño artístico como industrial.

Los lectores podrán encontrar información adicional sobre estos temas en el artículo de García, Pérez y Cocero (2007).

\subsection{Producción más limpia}

Si el proceso industrial no ha sido diseñado bajo los principios indicados, entonces se puede utilizar una estrategia conocida como Producción más limpia (PML) con la finalidad de contribuir al desarrollo sostenible. Igual que su "sinónimo" ecoeficiencia, la PML se define como una estrategia ambiental preventiva e integrada, enfocada hacia procesos productivos, productos y servicios, con la finalidad de reducir costos, incentivar innovaciones y disminuir los riesgos relevantes al ser humano y al ambiente.

Como estrategia, la PML puede tener aplicación en diferentes niveles de una misma industria, involucrando desde su misión hasta sus diferentes estrategias, sistemas, componentes, materiales y procesos. Sus alcances abarcan aspectos internos de la empresa industrial, como la calidad del producto, el acceso a tecnología alternativa, la disponibilidad de capital y la resistencia cultural; y aspectos externos como las políticas macroeconómicas y ambientales, aspectos financieros, la presión de la comunidad, la demanda en el mercado por productos sostenibles, y el acceso a tecnología alternativa.

La importancia de esta estrategia empresarial radica en su aporte a la competitividad empresarial basada en la conservación del medio ambiente y la responsabilidad social. De esta manera contribuye al equilibro entre los tres elementos principales del desarrollo sostenible como objetivo universal.

Los objetivos de la política de PML son los siguientes:
- Optimizar el consumo de recursos naturales y materias primas.

- Aumentar la eficiencia energética y utilizar combustibles más limpios.

- Prevenir la generación de residuos contaminantes.

- Prevenir, mitigar, corregir y compensar los impactos ambientales sobre la población y los ecosistemas.

- Adoptar tecnologías más limpias y prácticas de mejoramiento continuo de la gestión ambiental.

- Minimizar y aprovechar los residuos, en caso de que estos sean inevitables.

Los beneficios que se pueden obtener por la adopción de la PML se pueden dividir en:

A) Beneficios relacionados con el desarrollo del proceso:

- Reducción de los costos correspondientes a la materia prima utilizada.

- Procesos más eficientes y rendimientos comparativos mayores.

- Reducción de los costos en servicios.

- Reducción de los costos para el tratamiento de los residuos.

- Ingresos adicionales debido a los materiales reaprovechados.

- Menos costos asociados a las operaciones de mantenimiento.

- Disminución de accidentes industriales, laborales y enfermedades ocupacionales.

b) Beneficios relacionados con el desempeño de la empresa:

- Ahorro de dinero por el pago de multas y otros (compensaciones, indemnizaciones, etc.), debido a que la empresa cumple con regulaciones ambientales vigentes (normas ambientales específicas, como la ISO 14000).

- Cambio de la imagen pública de la empresa dentro de la comunidad, lo cual se apreciará en el aumento de las ventas, ya que los consumidores están cada vez más concienciados para comprar productos de empresas que no contaminen el ambiente.

- Reconocimiento y aceptación de los productos ofrecidos por la empresa en los mercados internacionales, no solo por su calidad sino por lo establecido en las normas internacionales. 


\section{PROBLEMAS AMBIENTALES}

Un problema ambiental es la alteración del equilibrio natural de los distintos componentes del medio ambiente (aire -de la atmósfera-, agua -de la hidrósfera-, suelo -de la litósfera-, población humana y ecosistemas - de la biosfera-). Los problemas ambientales pueden ser causados por fenómenos naturales, aquellos relacionados con la dinámica planetaria, o por las actividades humanas (ya sean extractivas, industriales o domésticas). En el primer caso, solo se pueden prevenir las consecuencias de los problemas ambientales no el problema en sí; mientras que en el segundo caso, los problemas ambientales se pueden evitar, controlar o corregir, pero sus consecuencias (efectos o impactos) no necesariamente se pueden mitigar [6].

Existen diversos criterios para clasificar los problemas ambientales:

\section{a) Según su origen:}

- Problemas ambientales de origen natural (contaminación del agua, aire y suelo debido a una erupción volcánica)

- Problemas ambientales de origen humano (antrópico o antropogénico) debido a actividades humanas (industriales o domésticas), tal como, el cambio climático debido al calentamiento global, generado por gases de efecto invernadero -GEIcomo el metano, $\mathrm{CH}_{4}$ ).

- Combinados (contaminación del suelo debido a vertimientos tóxicos, como resultado de lluvias intensas y el posterior colapso de un depósito de relaves).

b) Según el área de influencia de los impactos (efectos relevantes de una actividad industrial):

- Locales (ruido excesivo producido por la voladura de rocas en la extracción de minerales, a tajo abierto).

- Regionales (presencia de lluvia ácida en zonas alejadas de plantas químicas generadoras de gases ácidos, como el $\mathrm{SO}_{2}$ ).

- Globales (deterioro de la capa de ozono por las SAO -Sustancias agotadoras de ozono- como los halones).

Las causas de los problemas ambientales originados por actividades desarrolladas por la industria en general y la industria química en particular se pueden resumir en:
1. Producción, almacenamiento, transporte, consumo o aplicación de productos o subproductos que representan peligros para la población humana o para los ecosistemas. Tal es el caso de los combustibles usados en el transporte de materiales, el uso de explosivos en la extracción de minerales o los plaguicidas que son aplicados en el combate de plagas en los cultivos, entre otros.

2. Materias primas, insumos, productos, subproductos, residuos o desechos de un proceso químico industrial que tiene efectos negativos sobre:

- Personal de la planta (deterioro de la salud de trabajadores que laboran en la producción o manipulación de agroquímicos).

- Población o ecosistema situado en el área de influencia de la planta (las emisiones de las chimeneas de fundiciones de plomo cerca de centros poblados o áreas de cultivo).

3. Accidente o desastre químico: emisiones tóxicas generadas por un accidente tecnológico (fuga de isocianato de metilo desde una planta química en Bhopal, India, 1984).

Los problemas ambientales preocupan:

- Por la contaminación ambiental que generan, así como, por sus efectos sobre la salud humana a corto, mediano y largo plazo.

- Por los daños a los ecosistemas ocasionados por las actividades extractivas o industriales.

- Por la pérdida de calidad de los recursos no renovables, renovables y ambientales [8].

- Por el agotamiento de los recursos (no renovables y renovables).

- Por la generación de conflictos socioambientales, tanto a nivel local, regional, nacional o internacional.

\section{IMPORTANCIA DEL MEDIO AMBIENTE PARA LA INDUSTRIA}

En general, una gran parte de la sociedad se ha beneficiado de los productos y el bienestar creado por la industria; pero durante años, el sector industrial químico no tuvo en cuenta los impactos negativos que sus actividades generaban en el medio ambiente (local, regional o global). En la actualidad, la industria es consciente que para sobrevivir en un mundo cada vez más informado con respecto a las causas de los problemas ambientales, son esenciales las buenas 
prácticas; el mejoramiento continuo; el diálogo con la comunidad, con los consumidores, con los medios de comunicación, con las autoridades sectoriales y locales (municipales), con los grupos ambientalistas, así como con las organizaciones no gubernamentales, entre otras. La industria ha comprendido que la inversión en investigación científica y tecnológica, y en especial aquella que trata de la relación entre los aspectos tecnológicos y los aspectos ambientales, genera innovaciones que contribuyen a aumentar la competitividad y la rentabilidad de las empresas que son socialmente responsables de sus actividades [1].

Según el Banco Mundial, el medio ambiente es el conjunto complejo de condiciones físicas, geográficas, biológicas, sociales, culturales y políticas que rodean a un individuo (u organismo) y que, en definitiva, determinan su forma y la naturaleza de su supervivencia.

En el presente documento, se entiende por industria al colectivo formado por empresas de un sector industrial determinado, por ejemplo, el sector industrial químico. Es así, que el ambiente es importante con relación a la industria por las siguientes razones:

- Exigencias del mercado donde se realizan transacciones comerciales entre los vendedores (ofertantes) y los compradores (demandantes o consumidores).

- Diferenciación con la competencia, ya que con la finalidad de captar a sus clientes los ofertantes tienen que diferenciarse de sus competidores.

- Exigencias de los clientes, ya que los demandantes tienen requerimientos ambientales que tienen que ser satisfechos por los ofertantes.

- Exigencias de la sociedad, ya que existen miembros de la comunidad, que aunque no demanden los productos ofrecidos por la industria, sí están preocupados por los impactos ambientales de muchos de ellos.

El ciudadano está cada vez más preocupado por los daños provocados en el ambiente como consecuencia de las actividades antrópicas, tanto domésticas como industriales. También, la opinión pública está cada vez más sensibilizada con relación a los perjuicios causados por catástrofes ambientales que se han producido en los últimos años, tanto de origen natural como de origen humano (por ejemplo, accidentes químicos).

Esta creciente preocupación ciudadana ha motivado una legislación cada vez más estricta por parte de las autoridades gubernamentales de todo el mundo, las cuales vigilan cada vez más el cumplimiento de la normatividad ambiental, lo que trae como consecuencia "amenazas tangibles" (demandas legales, multas, etc.) para las empresas "sucias"; es decir, aquellas empresas que contaminan el ambiente, no solo por la cantidad de residuos y desechos que generan, sino por la inadecuada gestión de los mismos. Integrar la variable ambiental en la actividad industrial debe significar notables ventajas en la competitividad de las empresas, lo que implica conseguir a través del tiempo mejora de los resultados, condiciones ventajosas para competir en mercados cada vez más exigentes, bases tecnológicas limpias e innovadoras y actividades económicas sostenibles en el tiempo.

El medio ambiente puede crear en la práctica las condiciones para cumplir con estos requisitos, ya que es un hecho que la empresa que dispone de tecnologías para la protección ambiental y productos o servicios ambientalmente superiores, ya tiene una ventaja comparativa inicial. Además, la empresa que introduce mejoras en la organización y la gestión en el sistema de producción con la finalidad de utilizar tecnologías limpias (como parte de su estrategia empresarial conocida como producción limpia o producción más limpia) obtiene un elevado nivel de protección ambiental; lo cual le confiere una imagen que facilita la integración de la empresa en la comunidad en la que está establecida y predispone favorablemente a los consumidores para la preferencia por sus productos, lo cual se ve reforzado mediante una certificación internacional, tal como la ISO 14000.

\section{LA IMPORTANCIA DE LOS RECURSOS NATURALES COMO MATERIA PRIMA}

Los procesos productivos requieren de materia prima, la cual proviene de los recursos naturales que forman parte del medio ambiente. El término recurso natural resulta fácil de entender intuitivamente, pero sin embargo es muy difícil de conceptuar y formalizar rigurosamente. Si se intenta definir los recursos naturales con base a sus propiedades físicas, se puede indicar que los recursos naturales son factores que afectan los procesos de producción y consumo, tienen su origen en fenómenos o procesos naturales que escapan al control del hombre. Los procesos naturales de generación del recurso pueden ser biológicos, geológicos o químicos. Estos procesos generadores pueden ser muy cortos (por ejemplo, proceso de formación del agua de lluvia) o de muy 
larga duración (por ejemplo, proceso de formación de una "bolsa" de petróleo).

Si se intenta definir los recursos desde un punto de vista económico, se puede decir que los recursos naturales son factores que afectan las actividades productivas, pero que no han sido elaborados por el hombre, ni tampoco han sido obtenidos a través de un proceso de fabricación iniciado por el hombre.

Las dificultades que se encuentran al tratar de definir un recurso natural son mayores cuando se intenta establecer una clasificación de los mismos, según el criterio que se elija se encontrará una definición diferente [8].

- Por la estructura material del recurso, se pueden tener:

a) Biológicos (pesquerías, bosques, etc.).

b) Minerales (metálicos y no metálicos).

c) Energéticos (petróleo, gas natural, carbón).

d) Ambientales (aire, capa de ozono, paisajes, etc.).

- Por el marco temporal se tienen:

a. Recursos no renovables, cuando la utilización (consumo) de una unidad del recurso implica su completa destrucción, abarcando su regeneración periodos de tiempo muy grandes, tales como el carbón, el petróleo, etc.

b. Recursos no renovables con servicios reciclables, cuando el uso del recurso implica su completa destrucción en cuanto a su forma actual, pero es recuperable en un futuro más o menos inmediato por medio de un proceso industrial de reciclado (los recursos minerales: hierro, aluminio, cobre, etc.).

c. Recursos renovables, cuando el uso del recurso produce su agotamiento o destrucción, pero seguidamente se produce la regeneración del mismo según un mecanismo de naturaleza biológica (las pesquerías, los bosques, etc.).

d. Recursos ambientales, cuando el uso no implica su agotamiento o en caso de agotarse, su velocidad de reproducción o regeneración es rápida (agua, aire, paisaje, etc.).

\section{EL PAPEL DE LA INGENIERÍA DE PROCESOS EN EL LOGRO DEL DESARROLLO SOSTENIBLE}

La ingeniería de procesos es una especialidad tecnológica que, aplicando los principios fundamentales de la ingeniería, economía y ecología, busca producir bienes (o productos) que la sociedad requiere para satisfacer sus necesidades. El proceso productivo corresponde al componente operativo de la empresa, la cual para ser competitiva debe buscar producir óptimamente, es decir, con eficiencia tecnológica, organizacional, económica, social y ambiental.

Los ingenieros de procesos diseñan, operan y controlan procesos industriales (o procesos químicos tecnológicos), en los cuales se utilizan y transforman materias primas (recursos naturales) o sustancias químicas (resultantes de otros procesos), en productos o insumos. Actualmente el nuevo paradigma de los ingenieros de procesos se centra en el diseño y operación de procesos industriales sostenibles.

\section{PROCESO INDUSTRIAL SOSTENIBLE PARA LA PRODUCCIÓN DE PULPA DE CELULOSA BLANQUEADA}

Como un ejemplo de un proceso industrial sostenible que se aplica actualmente, se tiene el caso de la producción de pulpa de celulosa blanqueada (o pasta papelera). El proceso en la planta se inicia con el astillado de la madera, las astillas obtenidas se cocinan con un "licor blanco" (una mezcla de hidróxido y sulfuro de sodio). En la cocción se separa la celulosa (pulpa) de la lignina (que luego habrá de ser quemada). Se bombea la pulpa y la suspensión química residual para su depuración y deslignificación con oxígeno. Luego las fibras pasan al blanqueo y secado. Por su parte, la lignina y otros compuestos (hemicelulosas) que quedan en el licor resultante ("licor negro"), se concentran (evaporan) hasta llegar a un $75 \%$ de sólidos secos. El "licor negro" concentrado se quema en una caldera de recuperación para producir energía, mientras que las sustancias químicas se reciclan para ser usadas otra vez en el proceso de cocción. El proceso, no solamente es autosuficiente en el aspecto energético y químico, sino que además está diseñado para producir un excedente de bioenergía. El fundido inorgánico "licor verde" es caustificado 
Figura 2. Proceso industrial sostenible para la producción de pulpa blanqueada $(R=$ residuo)

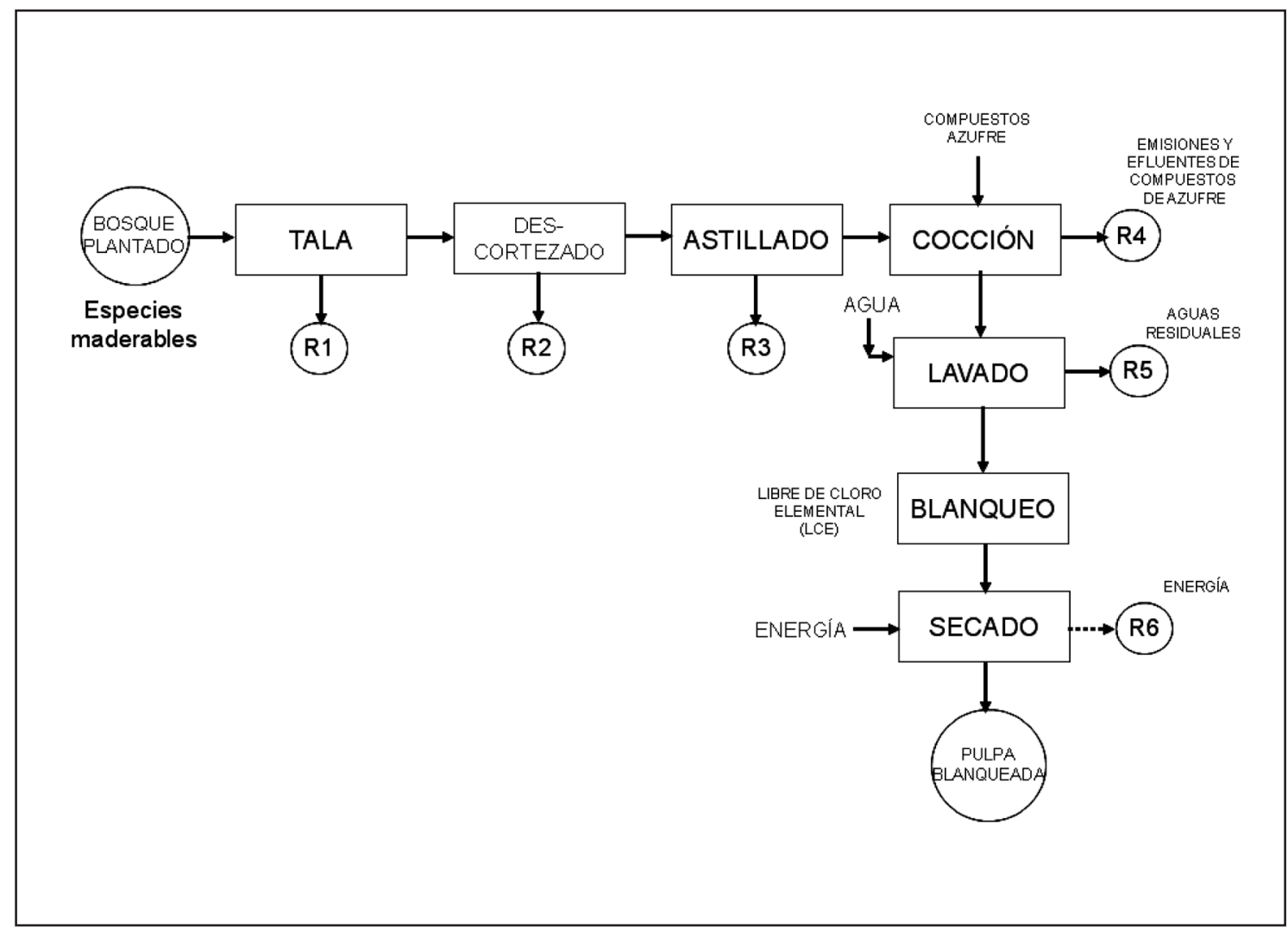

Fuente: Elaboración propia, 2013.

por medio del agregado de cal produciéndose nuevamente "licor blanco" para su reutilización en el digestor de cocción, y lodo de cal. El lodo de cal se calcina en un horno para recuperar la cal que también es reutilizada. Como consecuencia de usar la materia prima de forma eficiente, queda muy poco residuo sólido para ser desechado en un vertedero (menos del $1 \%$ de la materia prima inicial). La pulpa depurada y deslignificada es enviada a la etapa de blanqueo mediante el cual se logra eliminar toda la lignina residual posible de la pulpa mediante el uso de productos químicos. En el blanqueo LCE (Libre de Cloro Elemental), las sustancias químicas principales son dióxido de cloro, soda cáustica (y/o licor blanco oxidado), oxígeno, peróxido de hidrógeno y ácido sulfúrico. Una planta con este proceso puede producir 1 $000000 \mathrm{ADt} / \mathrm{a}$ (toneladas secas al aire) de pulpa de eucalipto con una blancura de $89-92 \%$ según normas ISO. Una planta con esta tecnología está operando actualmente en Uruguay y podría servir como base para la producción sostenible de pasta papelera a nivel nacional.

\section{CONCLUSIONES}

- Las empresas responsables y competitivas, que transforman la materia prima y la energía en productos $\mathrm{o}$ insumos, tienen en la planta industrial el componente operativo de la organización. En la planta industrial se lleva a cabo un proceso, el cual debe ser sostenible.

- El proceso industrial sostenible es innovador, eficiente, "limpio", seguro y que contribuyen al Desarrollo Sostenible.

- Los procesos industriales sostenibles utilizados por empresas responsables y competitivas, para elaborar productos químicos en particular, deben ser innovadores; es decir, deben incorporar en su diseño etapas que sean eficientes, que generen menos residuos y desechos, que no contaminen el ambiente, que utilicen sustancias que sean inocuas, así como los productos y subproductos elaborados; que sean seguras internamente (para los trabajadores) y externamente (para las comunidades y ecosistemas) y que contribuyan al desarrollo sostenible, garantizando el uso 
eficiente de recursos naturales, propiciando el empleo de recursos renovables.

- Para el diseño de procesos industriales sostenibles es importante emplear los fundamentos de la Química Verde (con los aportes del concepto de la síntesis ideal), la Ingeniería Verde, la estrategia integrada de la cuna a la cuna y la Biomimética.

- Si la empresa ya está operando se debe aplicar una estrategia empresarial preventiva como la Producción más Limpia, tanto en sus aspectos organizativos como en sus aspectos operativos (tecnologías limpias).

- Una empresa que utiliza en su planta un proceso industrial sostenible, no debe generar problemas ambientales.

- Tomando como referencia el proceso presentado, a nivel nacional se podrían utilizar los "bosques sembrados" de eucalipto para la obtención de pulpa de celulosa blanqueada, mediante un proceso industrial sostenible, que cumpla con las características de este tipo de procesos.

\section{REFERENCIAS BILIOGRÁFICAS}

[1] Alfaro de La Torre Juan, Olcese Aldo, Rodríguez Miguel Ángel (2008). Manual de la empresa responsable y sostenible. Conceptos y herramientas de la Responsabilidad Social Corporativa o de la Empresa. 1 $^{\text {a }}$. ed. McGraw-
Hill Interamericana de España S.A. Madrid. 2008.

[2] Considine Timothy (s/f). Ecología Industrial. Pennsylvania State University. Estados Unidos. Fuente: www.mideplan.go.cr/sinades/ PUBLICACIONES/ (Visitado el 13-08-09).

[3] García, J., Pérez, L., Cocero, M.J. (2007). Nuevas bases para el diseño de procesos industriales sostenibles. Ingeniería Química; XXXIX (444), 106-113.

[4] Gómez Cívicos J.I. (2008). Ingeniería Verde: Doce principios para la sostenibilidad. Ingeniería Química; XL (458), 168-175.

[5] Loayza Jorge (2006). Parques Industriales Ecoeficientes. Revista Electrónica ECOMUNDO N. ${ }^{\circ}$ 11. Octubre. 2006. www.revistaecomundo. com. (Visitado el 08-12-12).

[6] Loayza Jorge (2012). Procesos industriales Sostenibles Conferencia. ECli-2012. Encuentro Científico Internacional de Invierno. Lima.

[7] Pellerin Cheryl (s/f) "La Química Verde". OPII del Departamento de Estado de EEUU. Fuente: http://usinfo.state.gov/journals/itgic/0605/ijgs/ pellerin.htm. (Visitado el 20-07-11)

[8] Romero Carlos (1997). Economía de los Recursos Ambientales y Naturales. 2. ${ }^{\text {a }}$ ed. Ampliada. Alianza Editorial. Madrid.

[9] Spiro Thomas G., Stigliani William M. (2004). Química Medioambiental. 2. ${ }^{\text {a }}$ ed. Pearson Educación S.A. Madrid. 